PSU-TH-180

December 1996

\title{
SEVEN SPHERE AND THE EXCEPTIONAL NONLINEAR SUPERCONFORMAL ALGEBRAS
}

\author{
Murat Günaydin 1 \\ Physics Department, 104 Davey Lab. \\ Penn State University \\ University Park, PA 16802, U.S.A. \\ Talk given at the 30th International Symposium \\ on the Theory of Elementary Particles, \\ Buckow, Germany (August 27-31, 1996).
}

\begin{abstract}
The realizations of the exceptional non-linear (quadratically generated, or $W$ type) $N=8$ and $N=7$ superconformal algebras with $\operatorname{Spin}(7)$ and $G_{2}$ affine symmetry currents are reviewed. Both the $N=8$ and $N=7$ algebras admit unitary realizations in terms of a single boson and free fermions in $\underline{8}$ of $\operatorname{Spin}(7)$ and $\underline{7}$ of $G_{2}$, with the central charges $c_{8}=26 / 5$ and $c_{7}=5$, respectively. They can also be realized over the coset spaces $S O(8) \times U(1) / S O(7)$ and $S O(7) \times U(1) / G_{2}$ for some fixed values of their central charges, respectively. The coset space $S O(8) / S O(7)$ is the seven-sphere $S^{7}$, whereas the space $S O(7) / G_{2}$ represents the seven-sphere with torsion. We conclude with a discussion of a novel 'hybrid' method developed recently that yields unitary realizations of the exceptional $N=8$ and $N=7$ algebras for all allowed values of their central charges.
\end{abstract}

\footnotetext{
${ }^{1}$ Work supported in part by the National Science Foundation under research Grant No. PHY-9631332.

email: murat@phys.psu.edu
} 


\section{Introduction}

The $N$-extended quadratically non-linear superconformal algebras of the type introduced by Bershadsky and Knizhnik 11, 2] involve generators of conformal dimension 2, 3/2 and 1 only. The anticommutators of the supersymmetry generators of these algebras close into the Virasoro generators, generators of the symmetry currents of dimension one and their bilinears. A complete classification of complex forms of simple reductive non-linear superconformal algebras was given in refs. [3, 任. Reductivity means that these algebras linearise in the limit of infinite central charge. In this limit, the infinite-dimensional vacuum-preserving algebra becomes a finite superalgebra containing the finite (global) conformal algebra and the classification of such non-linear superconformal algebras [3, 4] reduces to the classification of finite-dimensional (global) superconformal algebras given in ref. [5]. There are three infinite classical families (for either the right- or the left-moving modes),

$$
\operatorname{osp}(N \mid 2 ; \mathbf{R}), \quad \operatorname{su}(1,1 \mid N), \quad \operatorname{osp}\left(4^{*} \mid 2 N\right)
$$

,a one-parameter family of $N=4$ algebras, and two exceptional superconformal algebras with $N=7$ and $N=8$ supersymmetries. The vacuum-preserving subalgebras of the $N=7$ and $N=8$ exceptional superconformal algebras in the limit of infinite central charge are the exceptional finite Lie superalgebras $G(3)$ and $F(4)$ (in Kač's notation [6]), respectively. 2 In this talk I will review the realizations of the exceptional nonlinear superconformal algebras. Following my joint work with Sergei Ketov [10] I will first give their realizations in terms of a single boson and $N$ fermions and then study their realizations over the coset spaces $S O(8) \times U(1) / S O(7)$ and $S O(7) \times U(1) / G_{2}$, respectively. Both of these realizations give some definite values for the central charges of the respective algebras. I will conclude with the discussion of their realization via a "hybrid" method given recently in my joint work with Behzad Bina [11. This hybrid method involves fermions in the coset of some supergroup, a scalar field (dilaton) and dimension one currents corresponding to the symmetries of these algebras and yields realizations for all allowed values of the central charge determined by the level of the symmetry current algebra.

For discussion of physical motivations, possible applications to string theory as well as further details of the results reported below and further references we refer to references [10] and [11].

\footnotetext{
${ }^{2}$ See refs. $[7,8$ for details about the $G(3)$ and $F(4)$. Another real form of $F(4)$ corresponds to $N=2$ superconformal symmetry in five space-time dimensions 9 .
} 


\section{Exceptional $N=7$ and $N=8$ Superconformal Algebras}

It is most convenient to represent the commutation and anticommutation relations [4] of the generators of the $N=8$ and $N=7$ non-linear superconformal algebras as operator products of chiral fields ( either in the left or the right moving sector) defined in terms of these generators [10]. These algebras can be obtained either via a Drinfeld-Sokolov-type reduction from affine versions of the exceptional Lie superalgebras $F(4)$ and $G(3)$, respectively 12 , or by purely algebraic methods [3, 4 . The $N=8$ algebra contains eight supercurrents $S^{M}$ of conformal dimension $3 / 2$, and 21 symmetry currents of $\operatorname{Spin}(7)$ under which the supercurrents transform in the spinor representation. The $N=7$ algebra has 7 supercurrents and 14 symmetry currents of $G_{2}$. Because of their non-linearity, the 'vacuum-preserving' algebra, generated by the modes $S_{ \pm 1 / 2}^{M}$ is not finite. However in the limit of infinite central charge it becomes finite.

\subsection{Exceptional $N=8$ superconformal algebra}

The bosonic part of the $N=8$ algebra is a semi-direct sum of the affine algebra $\widehat{s o(7})_{k}$ of level $k$ and the Virasoro algebra. Their OPEs are given as

$$
\begin{aligned}
T(z) T(w) & \sim \frac{c / 2}{(z-w)^{4}}+\frac{2 T(w)}{(z-w)^{2}}+\frac{\partial T(w)}{z-w} \\
T(z) T^{m n}(w) & \sim \frac{T^{m n}(w)}{(z-w)^{2}}+\frac{\partial T^{m n}(w)}{z-w} \\
T^{m n}(z) T^{p q}(w) & \sim \frac{-i}{z-w}\left\{\delta^{n p} T^{m q}(w)+\delta^{m q} T^{n p}(w)-\delta^{m p} T^{n q}(w)(2-2)\right. \\
& \left.-\delta^{n q} T^{m p}(w)\right\}+\frac{k}{(z-w)^{2}}\left\{\delta^{m p} \delta^{n q}-\delta^{m q} \delta^{n p}\right\}
\end{aligned}
$$

where the adjoint representation of $S O(7)$ is labeled by a pair of antisymmetric indices, $m, n, \ldots=1,2, \ldots, 7$. Since the supercurrents $S^{M}(z)$ transform in the spinor representation of $\operatorname{Spin}(7)$ and have spin $3 / 2$, we have

$$
\begin{aligned}
& T(z) S^{M}(w) \sim \frac{\frac{3}{2} S^{M}(w)}{(z-w)^{2}}+\frac{\partial S^{M}(w)}{z-w} \\
& T^{m n}(z) S^{M}(w) \sim \frac{-i}{2} \frac{\gamma_{M N}^{m n}}{(z-w)} S^{N}(w)
\end{aligned}
$$

where $\gamma^{m n}=\frac{1}{2}\left[\gamma^{m}, \gamma^{n}\right]$ with $\gamma^{m}$ the $8 \times 8$ gamma matrices in seven dimensions. These gamma matrices can be written in terms of the octonionic 
structure constants 13, 14, 15, 16, 10. 3 The non-trivial OPE's are the ones corresponding to the products of $N=8$ supersymmetry generators which read as follows:

$$
\begin{aligned}
S^{M}(z) S^{N}(w) \sim & \frac{8 k(k+2)}{3(k+4)} \frac{\delta^{M N}}{(z-w)^{3}}+\frac{2 T(w)}{z-w} \delta^{M N} \quad(2-4) \\
& -\frac{\delta^{M N}}{3(k+4)} \frac{T^{m n} T^{m n}(w)}{z-w}-\frac{1}{12(k+4)} \gamma_{m n p q}^{M N} \frac{: T^{m n} T^{p q}:(w)}{z-w} \\
& +\frac{2 i(k+2)}{3(k+4)} \gamma_{m n}^{M N}\left\{\frac{T^{m n}(w)}{(z-w)^{2}}+\frac{\partial T^{m n}(w)}{2(z-w)}\right\}
\end{aligned}
$$

where $M, N=1, \ldots, 8$, and $m, n \ldots=1, \ldots, 7$.

The Jacobi 'identities' require that the central charge $c$ of the $N=8$ algebra

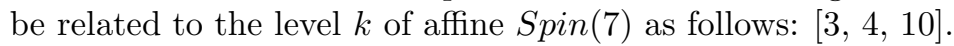

$$
c=c_{8} \equiv 4 k+\frac{6 k}{k+4} \equiv \frac{2 k(2 k+11)}{k+4},
$$

\subsection{Exceptional $N=7$ superconformal algebra}

The exceptional $N=7$ non-linear superconformal algebra is similar to the $N=8$ algebra having the gauge group $G_{2}$ instead of $\operatorname{Spin}(7)$ and seven supercurrents. We shall denote the generators of $G_{2}$ as $G^{A}$ and the seven-dimensional representation matrices of $G_{2}$ as $M^{A}(A=1,2, \ldots, 14)$. The matrices $M^{A}$ satisfy the identities [13, 田, 10]

$$
\begin{aligned}
& \operatorname{tr}\left(M^{A} M^{B}\right)=2 \delta^{A B} \\
& M_{i j}^{A} M_{k l}^{A}=\frac{2}{3}\left(\delta_{i l} \delta_{j k}-\delta_{i k} \delta_{j l}\right)-\frac{1}{3} C_{i j k l}
\end{aligned}
$$

where $C_{i j k l}$ is the $G_{2}$ invariant completely antisymmetric tensor . The seven supercurrents $S^{i}(z)$ transform in 7 of $G_{2}$, and satisfy the OPEs

$$
\begin{aligned}
T(z) S^{i}(w) & \sim \frac{\frac{3}{2} S^{i}(w)}{(z-w)^{2}}+\frac{\partial S^{i}(w)}{z-w} \\
G^{A}(z) S^{i}(w) & \sim \frac{1}{z-w} M_{i j}^{A} S^{j}(w)
\end{aligned}
$$

The most important OPE's are again the ones between the $N=7$ supersymmetry generators

\footnotetext{
${ }^{3}$ There exists two different realizations of the gamma matrices in seven dimensions in terms of the octonionic structure constants. We shall denote them as $\gamma^{m}$ and $\tilde{\gamma}^{m}$ 1ㅐ. The antisymmetric products of gamma matrices are defined with unit weight, viz. $\gamma^{i j \cdots k}=\gamma^{[i} \gamma^{j} \cdots \gamma^{k]}$.
} 


$$
\begin{aligned}
S^{i}(z) S^{j}(w) \sim & \frac{k(3 k+5)}{k+3} \frac{\delta^{i j}}{(z-w)^{3}}+\frac{3 k+5}{k+3} M_{i j}^{A}\left\{\frac{G^{A}(w)}{(z-w)^{2}}+\frac{1}{2} \frac{\partial G^{A}(w)}{z-w}\right\} \\
& +\frac{\delta^{i j}}{z-w}\left\{2 T(w)-\frac{1}{k+3} G^{A} G^{A}(w)\right\} \\
& +\frac{3}{4(k+3)} \frac{\left\{M^{A} M^{B}+M^{B} M^{A}\right\}^{i j} G^{A} G^{B}(w)}{(z-w)}
\end{aligned}
$$

The central charge is given by [3, 4, 10]

$$
c=c_{7} \equiv \frac{9}{2} k+\frac{2 k}{k+3} \equiv \frac{k(9 k+31)}{2(k+3)}
$$

\section{Coset Space Realizations}

The structure of the exceptional nonlinear $N=8$ and $N=7$ superconformal algebras is such that they do not admit realizations over an infinite family of coset spaces $G / H$ in contrast to superconformal algebras with $N \leq 4$ [10. Starting with a most general Ansatz for a coset space realization of these algebras one finds that such a realization is possible for a unique compact coset space for each of the exceptional algebras [10], namely $S O(8) \times U(1) / S O(7)$ for the $N=8$ algebra and $S O(7) \times U(1) / G_{2}$ for the $N=7$ algebra.

\subsection{A construction of the exceptional $N=8$ superconfor- mal algebra over the coset space $S O(8) \times U(1) / S O(7)$}

Consider the affine algebra $\widehat{s o(8)})_{\hat{k}} \oplus \widehat{u(1)}$, defined by the OPEs

$$
\begin{aligned}
\hat{J}^{a b}(z) \hat{J}^{c d}(w) \sim & \frac{2}{z-w}\left\{\delta^{b c} \hat{J}^{a d}(w)+\delta^{a d} J^{b c}(w)-\delta^{a c} \hat{J}^{b d}(w)-\delta^{b d} \hat{J}^{a c}(w)\right\} \\
& -\frac{4 \hat{k}}{(z-w)^{2}}\left\{\delta^{a c} \delta^{b d}-\delta^{a d} \delta^{b c}\right\}
\end{aligned}
$$

and

$$
\hat{J}^{8}(z) \hat{J}^{8}(w) \sim \frac{\hat{k}_{1} / 2}{(z-w)^{2}}
$$

where $a, b, \ldots=1,2, \ldots, 8$. The $\hat{k}_{1}$ is a normalisation parameter of the $U(1)$ current $\hat{J}^{8}$. The currents $\hat{J}^{m}= \pm i \hat{J}^{m 8}, m=1, \ldots, 7$, belonging to the coset $S O(8) / S O(7)$ satisfy the OPE

$$
\hat{J}^{m}(z) \hat{J}^{n}(w)=\frac{4 \hat{k}}{(z-w)^{2}} \delta^{m n}+\frac{2 \hat{J}^{m n}(w)}{z-w}+\hat{J}^{m} \hat{J}^{n}(w)+\ldots
$$


Associated with the currents $\hat{J}^{a}=\left(\hat{J}^{m}, \hat{J}^{8}\right)$ of the coset $S O(8) \times U(1) / S O(7)$ we introduce free fermionic fields $\psi^{a}=\left(\psi^{m}, \psi^{8}\right), a, b, \ldots=1,2, \ldots, 8$

$$
\psi^{a}(z) \psi^{b}(w)=\frac{1 / 2 \delta^{a b}}{z-w}+(z-w) \partial \psi^{a} \psi^{b}(w)
$$

One finds that a consistent solution to all the contraints exists and leads to the following expressions for the generators of the $N=8$ algebra in terms of the coset space currents and fermions 10

$$
\begin{aligned}
& T^{m n}=-\frac{i}{2}\left\{\hat{J}^{m n}+\bar{\psi} \tilde{\gamma}^{m n} \psi\right\} \\
& S^{m}=i \sqrt{\frac{7}{33}} \gamma_{a b}^{m} \psi^{a} \hat{J}^{b}, \quad S^{8}=\sqrt{\frac{7}{33}} \psi^{a} \hat{J}^{a} \\
& T=\frac{1}{132}\left\{7 \hat{J}^{a} \hat{J}^{a}-\hat{J}^{m n} \hat{J}^{m n}-42 \bar{\psi} \partial \psi+\frac{3}{2} \hat{J}^{m n}\left(\bar{\psi} \tilde{\gamma}^{m n} \psi\right)-\left(\bar{\psi} \tilde{\gamma}^{m n} \psi\right)\left(\bar{\psi} \tilde{\gamma}^{m n} \psi\right)\right\}
\end{aligned}
$$

with central charge $c=84 / 11$ corresponding to the level $k=\hat{k}+1=3 / 2$.

\subsection{A construction of the exceptional $N=7$ superconfor- mal algebra over the coset space $S O(7) \times U(1) / G_{2}$}

The automorphism group $G_{2}$ of octonions is a 14-dimensional subgroup of $S O(7)$. Under $G_{2}$, the adjoint representation of $\operatorname{Spin}(7)$ decomposes as $21=$ $14+7$. One can choose a basis for $G_{2}$ such that its generators $G^{i j}$ can be expressed in terms of the generators $J^{i j}$ of $S O(7)$ in a simple form [15, 17, 10]:

$$
G^{i j}=\frac{1}{2} J^{i j}+\frac{1}{8} C^{i j}{ }_{k l} J^{k l}
$$

The $G^{i j}$ are not all linearly independent and satisfy the constraints

$$
C_{i j k} G^{j k}=0
$$

where $C_{i j k}$ are the completely antisymmetric structure constants of the octonions. The remaining seven generators of $S O(7)$ can be chosen as

$$
A^{i}=\frac{1}{2} C^{i j k} J^{j k}
$$

They are associated with the seven-dimensional coset space $S O(7) / G_{2}$. Hence we have the decomposition [17]

$$
J^{i j}=\frac{4}{3} G^{i j}+\frac{1}{3} C^{i j k} A^{k}
$$


Note that the coset space $S O(7) / G_{2}$ is not a symmetric space. The symmetric space $S O(8) / S O(7)$ can be identified with the round seven-sphere $S^{7}$ and the space $S O(7) / G_{2}$ as the seven-sphere with torsion.

For the $N=7$ algebra we start with the affine algebra $\widehat{S O(7)_{\hat{k}}}$. Denoting the currents associated with the coset space $S O(7) / G_{2}$ and the group $G_{2}$ as $\hat{A}^{m}(z)$ and $\hat{G}^{m n}(z)$, respectively, we have

$$
\begin{aligned}
& \hat{A}^{m}(z)=\frac{1}{2} C_{n p}^{m} \hat{J}^{n p}(z) \\
& \hat{G}^{m n}(z) \equiv \frac{1}{2} \hat{J}^{m n}(z)+\frac{1}{8} C^{m n} \quad p q \hat{J}^{p q}(z)
\end{aligned}
$$

The coset space currents satisfy the OPE

$$
\hat{A}^{m}(z) \hat{A}^{n}(w)=\frac{-12 \hat{k}}{(z-w)^{2}} \delta^{m n}+\frac{1}{(z-w)}\left\{2 C_{k}^{m n} \hat{A}^{k}-8 \hat{G}^{m n}\right\}
$$

We shall denote the $\widehat{U(1)}$ current as $\hat{A}^{0}(z)$

$$
\hat{A}^{0}(z) \hat{A}^{0}(w)=\frac{1 / 2}{(z-w)^{2}}+\hat{A}^{0} \hat{A}^{0}(w)
$$

and the $8=1+7$ free fermions associated with the coset $S O(7) \times U(1) / G_{2}$ as $\psi^{a}(z)$ whose OPE's are the same as for the $N=8$ algebra.

Again one finds that there exists a unique solution to all the constraints of the $N=7$ algebra which yields the following expressions for its generators 10]

$$
\begin{aligned}
G^{A}= & -\frac{1}{4}\left(M^{A}\right)^{m n}\left\{\hat{G}^{m n}+\bar{\psi} g^{m n} \psi\right\} \\
S^{m}= & \frac{i}{6} \sqrt{\frac{19}{6}}\left\{C_{n p}^{m} \psi^{n} \hat{A}^{p}+6 i \psi^{m} \hat{A}^{0}+3 \partial \psi^{m}\right\} \\
T= & -\frac{1}{84}\left(\hat{G}^{m n}+\bar{\psi} g^{m n} \psi\right)^{2}-\frac{19}{7 \cdot 12^{2}}\left\{\hat{A}^{m} \hat{A}^{m}-42 \hat{A}^{0} \hat{A}^{0}\right. \\
& \left.+21 i \partial \hat{A}^{0}+18 \psi^{m} \partial \psi^{m}-16 \hat{G}^{m n}\left(\bar{\psi} g^{m n} \psi\right)+12 i \hat{A}^{m}\left(\bar{\psi} \tilde{\gamma}^{m} \psi\right)\right\}
\end{aligned}
$$

where $g^{m n}=\frac{1}{2} \gamma^{m n}+\frac{1}{8} C_{p q}^{m n} \gamma^{p q}$ and $\psi^{8}=0$. The $M^{A}$ are the seven-dimensional representation matrices of $G_{2}, A=1,2, \ldots, 14$, providing the explicit embedding of $G_{2}$ into $S O(7)$ [13, [4, 10]. They satisfy the identities [4, 10]

$$
\begin{aligned}
& \operatorname{tr}\left(M^{A} M^{B}\right)=2 \delta^{A B} \\
& M_{i j}^{A} M_{k l}^{A}=\frac{2}{3}\left(\delta_{i l} \delta_{j k}-\delta_{i k} \delta_{j l}\right)-\frac{1}{3} C_{i j k l}
\end{aligned}
$$


It should be noted that the currents $\hat{A}^{m}$ are anti-hermitian which leads to factors of $i$ in the expressions for $S^{m}$ and $T$ so as to make all the terms appearing in them hermitian as required by unitarity. The above realization of the exceptional nonlinear $N=7$ algebra has central charge $c=89 / 12$ corresponding to $k=\hat{k}+1=3 / 2$.

\subsection{Realization in Terms of a Single Boson and $N$ Free Fermions}

In addition to the coset space solution given above to the most general Ansatz for the realization of the exceptional nonlinear superconformal algebras there exist another solution for each of these algebras in terms of a single boson and $N$ free fermions. 10 For the $N=8$ algebra this realization leads to the following expressions for the generators 10 .

$$
\begin{aligned}
T^{m n} & =-\frac{i}{2}\left(\bar{\psi} \tilde{\gamma}^{m n} \psi\right) \\
S^{m} & =\frac{i}{\sqrt{5}}\left(i \psi^{m} \hat{J}^{8}+\frac{1}{3} C_{n p q}^{m} \psi^{n} \psi^{p} \psi^{q}+C_{p q}^{m} \psi^{p} \psi^{q} \psi^{8}\right) \\
S^{8} & =\frac{i}{\sqrt{5}}\left(-i \psi^{8} \hat{J}^{8}+C_{m n p} \psi^{m} \psi^{n} \psi^{p}\right) \\
T & =\frac{1}{20} \hat{J}^{8} \hat{J}^{8}-\frac{3}{8} \bar{\psi} \partial \psi+\frac{1}{240}\left(\bar{\psi} \tilde{\gamma}^{m n} \psi\right)\left(\bar{\psi} \tilde{\gamma}^{m n} \psi\right)
\end{aligned}
$$

with the central charge $c=26 / 5$ and level $k=1$ of the affine symmetry $\widehat{\operatorname{Spin}}(7)$.

For the $N=7$ algebra the corresponding realization gives

$$
\begin{aligned}
G^{A} & =-\frac{1}{3}\left(M^{A}\right)^{m n}\left(\bar{\psi} g^{m n} \psi\right) \\
S^{m} & =\frac{2}{\sqrt{6}}\left\{2 \psi^{m} \hat{A}^{0}-\frac{i}{3} C_{n p q}^{m} \psi^{n} \psi^{p} \psi^{q}\right\} \\
T & =\frac{2}{3} \hat{A}^{0} \hat{A}^{0}-\frac{2}{3} \psi^{m} \partial \psi^{m}+\frac{1}{126}\left(\bar{\psi} g^{m n} \psi\right)\left(\bar{\psi} g^{m n} \psi\right)
\end{aligned}
$$

In this case the central charge is $c=5$ with the level $k=1$ of the affine symmetry $\hat{G}_{2}$. 


\section{An "Hybrid" Realization of the Exceptional Nonlinear Superconformal Algebras for Arbi- trary Values of the Central Charge}

The coset space method and the method of free fermions with a single boson discussed above lead to realizations with a single definite value of the central charge in each case. Recently in a joint work with Behzad Bina [11] we have developed a very general "hybrid" method for the unified realization of all nonlinear superconformal algebras, quasi-superconformal algebras and $Z_{2}$ graded nonlinear superconformal algebras with both bosonic and fermionic supersymmetry generators for arbitrary values of the central charge. In particular, this method yields realizations of the exceptional nonlinear superconformal algebras for all allowed values of the central charge. The term "hybrid" refers to the fact that in this method the susy generators involve free fermions in the coset of a supergroup, a scalar field ( dilaton) and currents that form an affine Lie algebra isomorphic to the symmetry currents of the nonlinear superconformal algebra. For this realization we have used the formulation of nonlinear (quasi) superconformal algebras as given in [3]. In the formulation of [3] the OPE of the supersymmetry generators take on the form

$$
\begin{aligned}
G^{\alpha}(z) G^{\beta}(w)= & \frac{b \delta^{\alpha \beta}}{(z-w)^{3}}+\frac{\sigma \lambda_{a}^{\alpha \beta} J^{a}(w)}{(z-w)^{2}}+\frac{\sigma}{2} \frac{\lambda_{a}^{\alpha \beta} \partial J^{a}(w)}{(z-w)} \\
& +\frac{2 \delta^{\alpha \beta} T(w)}{(z-w)}+\frac{\gamma P_{a b}^{\alpha \beta} J^{a} J^{b}(w)}{(z-w)}
\end{aligned}
$$

where $\left(\lambda_{a}\right)^{\alpha \beta}=\lambda_{a}^{\alpha \beta}(a, b, \ldots=1,2, \ldots, \operatorname{dim} G)$ are the matrices of the representation under which the supersymmetry generators transform. The $J^{a}$ are the symmetry currents ( $\operatorname{Spin}(7)$ and $G_{2}$ for the exceptional algebras). They satisfy

$$
\begin{aligned}
J^{a}(z) G^{\alpha}(w) & =\frac{-\lambda_{\beta}^{a, \alpha} G^{\beta}(w)}{(z-w)} \\
J^{a}(z) J^{b}(w) & =-\frac{1}{2} \frac{k \ell^{2} \eta^{a b}}{(z-w)^{2}}+\frac{f_{c}^{a b} J^{c}(w)}{(z-w)}
\end{aligned}
$$

where $\ell^{2}=4(6)$, and $\alpha, \beta, \ldots=1.2, \ldots, 8(7)$, for the $N=8(7)$ algebras ,respectively. For the $N=8$ algebra one finds that the supersymmetry generators are of the form [11]

$$
G_{\alpha}(z)=\frac{-2 i\left(k_{0}+4\right)}{\sqrt{3 k_{0}+15}} \partial \psi_{\alpha}(z)+\partial \phi \psi_{\alpha}(z)
$$




$$
+\frac{i}{\sqrt{3 k_{0}+15}}\left\{\lambda_{a, \alpha}{ }^{\beta} K^{a} \psi_{\beta}(z)+\frac{1}{3} \lambda_{a, \alpha}{ }^{\beta} \lambda^{a, \gamma \delta} \psi_{\gamma} \psi_{\delta} \psi_{\beta}(z)\right\}(4-3)
$$

where $K^{a}(z)$ are the currents of an affine $\operatorname{Spin}(7)$ symmetry algebra of level $k_{0}$ :

$$
K^{a}(z) K^{b}(w)=\frac{-\frac{1}{2} k_{0} \ell^{2} \eta^{a b}}{(z-w)^{2}}+\frac{f_{c}^{a b} K^{c}(w)}{(z-w)}
$$

and $\psi^{\alpha}$ are eight free fermions

$$
\psi^{\alpha}(z) \psi^{\beta}(w)=\frac{\delta^{\alpha \beta}}{(z-w)}
$$

The scalar field $\phi(z)$ is normalized such that

$$
\partial \phi(z) \partial \phi(w)=\frac{1}{(z-w)^{2}}+\cdots .
$$

The central charge of the $N=8$ superconformal algebra generated by the above supersymmetry generators is

$$
c=\frac{\left(2 k_{0}+1\right)^{2}}{\left(k_{0}+5\right)}+\frac{21 k_{0}}{\left(k_{0}+5\right)}+5
$$

which agrees with the general expression for the central charge given in section 2 by noting that the 'full' level $k$ of the affine $S O(7)$ symmetry is $k=$ $k_{0}+1$.

For the $N=7$ algebra the hybrid realization gives the following expression for the supersymmetry generators [1]

$$
\begin{aligned}
G_{\alpha}(z)= & -\frac{i\left(3 k_{0}+10\right)}{\sqrt{6\left(k_{0}+4\right)}} \partial \psi_{\alpha}(z)+\partial \phi \psi_{\alpha}(z) \\
& +\frac{i}{\sqrt{6\left(k_{0}+4\right)}}\left\{\lambda_{a, \alpha}{ }^{\beta} K^{a} \psi_{\beta}(z)+\frac{1}{3} \lambda_{a, \alpha}{ }^{\beta} \lambda^{a, \gamma \delta} \psi_{\gamma} \psi_{\delta} \psi_{\beta}(z)\right\}(4-7)
\end{aligned}
$$

where now the $K^{a}(z)$ generate an affine $G_{2}$ of level $k_{0}$ and we have seven free fermions transforming in the 7 of $G_{2}$. The central charge of the resulting $N=7$ algebra is

$$
c=\frac{\left(3 k_{0}+2\right)^{2}}{2\left(k_{0}+4\right)}+\frac{14 k_{0}}{\left(k_{0}+4\right)}+\frac{9}{2}
$$

which again agrees with the general expression for the central charge given in section 2 by noting that $k=k_{0}+1$. 


\section{References}

[1] V. G. Knizhnik, Theor. Math. Phys. 66 (1986) 68.

[2] M. Bershadsky, Phys. Lett. 174B (1986) 285.

[3] E. Fradkin and V.YuLinetsky, Phys. Lett B275 (1992) 345 ; ibid B282 (1992) 352 .

[4] P. Bowcock, Nucl. Phys. B381 (1992) 415.

[5] M. Günaydin, G. Sierra and P. K. Townsend, Nucl. Phys. B274 (1986) 429.

[6] V. G. Kač, Infinite Dimensional Lie Algebras. An Introduction, Boston: Birkhäuser, 1983.

[7] M. Scheunert, W. Nahm and V. Rittenberg, J. Math. Phys. 17 (1976) 1626.

[8] B. de Witt and P. van Nieuwenhuizen, J. Math. Phys.23 1953.

[9] M. Günaydin, J. Math. Phys. 31 (1990) 1776; The Exceptional Superspace and the Quadratic Jordan Formulation of Quantum Mechanics, in "Elementary Particles and the Universe: Essays in Honor of Murray Gell-Mann" , edited by by J. H. Schwarz, Cambridge: Cambridge Univ. Press, 1991.

[10] M. Günaydin and S. Ketov, "Seven Sphere and the exceptional $N=7$ and $N=8$ Superconformal Algebras", Nucl. Phys. B467 (1996) 215

[11] B. Bina and M. Günaydin, "Real Forms of Nonlinear Superconformal and Quasi-superconformal Algebras and their Unified Realization" Penn State Preprint PSU-TH-172.

[12] K. Ito, J. O. Madsen and J. L. Petersen, Phys. Lett. 318B (1993) 315; Nucl. Phys. B398 (1993) 425.

[13] M. Günaydin and F. Gürsey, J. Math. Phys. 14 (1973) 1651.

[14] E. Corrigan, C. Devchand, D. B. Fairlie and J. Nuyts, Nucl. Phys. B214 (1983) 452.

[15] B.de Wit and H. Nicolai, Nucl. Phys. B231 (1984) 506.

[16] R.Dündarer, F.Gürsey and C.Tze, Nucl. Phys. B266 (1986) 440.

[17] M.Günaydin and H.Nicolai, Phys. Lett. 351B (1995) 169. 